\title{
Efeito da Usinagem na Rugosidade da Superfície da Madeira de Eucalyptus benthamii
}

\author{
Sabrina Andrade Martins ${ }^{1}$, Joana Mendes Ferraz ${ }^{1}$, \\ Cristiane Moreira Tavares dos Santos ${ }^{1}$, Cláudio Henrique Soares Del Menezzi², \\ Mário Rabelo de Souza ${ }^{3}$ \\ ${ }^{1}$ Programa de Pós-graduação em Ciências Florestais, Universidade de Brasília - UnB \\ ${ }^{2}$ Departamento de Engenharia Florestal, Universidade de Brasília - UnB \\ ${ }^{3}$ Laboratório de Produtos Florestais, Serviço Florestal Brasileiro
}

\begin{abstract}
RESUMO
O objetivo do presente trabalho foi analisar o efeito da usinagem feita por plaina e lixa na rugosidade da superfície da madeira de Eucalyptus benthamii Maiden et Cambage. A rugosidade da superfície foi analisada usando-se o rugosímetro Surftest SJ - 301. Imagens das superfícies obtidas por meio de um estereomicroscópio trinocular também foram analisadas. A rugosidade da superfície de E. benthamii não foi influenciada pelo modo de processamento da superfície (plaina ou lixa). Entretanto, foi possível observar pelas imagens que a superfície lixada apresentou fibrilações e ranhuras, enquanto a superfície aplainada apresentou células de vasos abertas.
\end{abstract}

Palavras-chave: qualidade, plaina, lixa.

\section{Machining Effect on the Surface Roughness of Eucalyptus benthamii Wood}

\begin{abstract}
The objective of this research was to evaluate the effect of planing and sanding on the surface roughness of Eucalyptus benthamii Maiden et Cambage wood. Surface roughness measurements were carried out using a Surftest SJ - 301 Profilometer. Surface images obtained by a trinocular stereo microscope were also analyzed. The E. benthamii surface roughness was not affected by the machining method (planing or sanding). However, it was possible to observe through the images that the sanded surface presented fibrillation and scratches, while the planed surface showed open vessel cells.
\end{abstract}

Keywords: quality, planer, sandpaper.

\section{INTRODUÇÃO}

A região do planalto catarinense apresenta um clima desfavorável para a plantação de eucalipto, sobretudo pela incidência de geadas. Entretanto algumas espécies como Eucalyptus dunnii e E. benthamii têm apresentado resistência significativamente superior comparada a outras espécies do gênero. A espécie E. benthamii foi plantada experimentalmente em Santa Catarina 
em 1988 pela Embrapa Florestas, a qual importou sementes da Austrália e obteve, a partir destas, uma área de produção de sementes. Já em 1992, a Epagri (Empresa de Pesquisa Agropecuária e Extensão Rural de Santa Catarina) iniciou o desenvolvimento do cultivo da espécie na região.

Em Santa Catarina o E. benthamii tem mostrado crescimento satisfatório e, como esperado, resistência à geadas em plantios experimentais (Higa \& Pereira, 2003). No estado do Paraná, na cidade de Colombo, árvores com 7 anos de idade apresentaram altura média de $21,7 \mathrm{~m}$ e diâmetro à altura do peito (DAP) médio de 18,2 cm (Pereira et al., 1997). Na cidade de Dois Vizinhos, também no Paraná, as árvores apresentaram altura média de 16 m e DAP médio de $15 \mathrm{~cm}$ aos 45 meses de idade; com isso os autores concluíram que a espécie merece atenção especial (Higa \& Carvalho, 1990).

Por essas vantagens apresentadas, a espécie está sendo inserida em plantios por órgãos de pesquisa e empresas madeireiras e papeleiras da região, provocando uma grande expectativa. Em decorrência disso se faz necessário o estudo dessa espécie, uma vez que poucas pesquisas foram realizadas em relação à qualidade da madeira, bem como sobre as propriedades tecnológicas apresentadas. Segundo Nisgoski et al. (1998), a madeira de E. benthamii apresenta como características gerais: densidade básica de $0,47 \mathrm{~g} / \mathrm{cm}^{3}$, sendo considerada moderadamente dura, cerne e alburno distintos, cerne de cor marrom- avermelhada e alburno amarelo-rosado, textura fina a média, grã irregular, cheiro e gosto imperceptíveis, superfície levemente brilhante em luz adequada, e anéis de crescimento indistintos. De acordo com Higa \& Pereira (2003), a madeira de E. benthamii é dimensionalmente instável, com índices elevados de contração volumétrica e de coeficiente de anisotropia, explicando a alta intensidade de defeitos observada na madeira serrada, mesmo em condições amenas de secagem. No entanto, os autores advertem que os estudos foram feitos com um pequeno número de amostras e, portanto, devem ser usados com ressalvas, sendo que futuros programas de melhoramento genético podem resolver em parte esses fatores restritivos.

A qualidade da superfície de produtos de madeira sólida é uma das propriedades mais importantes no processo de acabamento e na resistência de colagem (Kilic et al., 2006). As razões para preparar a superfície antes da colagem são: promover um contato adequado entre os aderentes e produzir uma superfície com corte recente (River et al., 1991). Segundo Piao et al. (2010), a umectação da superfície da madeira é dificultada ao longo do tempo através da contaminação, inativação ou envelhecimento.

De acordo com Silva et al. (2008), normalmente a qualificação da superfície usinada é feita por meio direto através da norma ASTM D1666-87 (1985), a qual apresenta um caráter subjetivo, ou por meio indireto através da determinação do avanço por dente (fz). Deste modo, o autor cita a necessidade de desenvolvimento de novas técnicas e/ ou equipamentos que analisem de forma precisa as superfícies usinadas da madeira.

Uma forma de avaliar a qualidade da superfície mais precisamente é por meio da medição da rugosidade, porém irregularidades superficiais em madeira maciça, tradicionalmente, não são medidas, tanto quanto para outros materiais engenheirados. Rugosímetros de agulha, perfilometria óptica, análise de imagens técnicas usando câmera de vídeo, pneumática, ultrassom, e microscopia são alguns dos métodos utilizados para avaliar a rugosidade superficial de produtos de madeira. No entanto, mesmo com a disponibilidade de todos esses métodos, não existe registro de uso frequente na indústria (Kilic et al., 2006).

A rugosidade da superfície da madeira é influenciada pela estrutura anatômica, particularmente as cavidades celulares (Taylor et al., 1999), associado a isso, o tipo de usinagem empregado durante o processamento também é responsável pela qualidade do produto final (Kilic et al., 2006).

Segundo Bonduelle et al. (2002), a usinagem da madeira pode ser expressa pela função $5 \mathrm{M}$, sendo:

- matéria-prima: relacionada com todas as propriedades intrínsecas da madeira e suas interações com o processo;

- máquina: relacionada com os aspectos construtivos das máquinas, incluindo os dispositivos de fixação, de condução e de exaustão de cavacos que visam melhoria da operação de usinagem;

- metodologia: engloba toda a definição dos parâmetros de usinagem a serem utilizados, bem como escolha das ferramentas mais adequadas 
para otimizar o processamento;

- mão de obra: considera o treinamento dos operadores executores das tarefas de usinagem, que devem ter conhecimentos de todos os itens anteriores para poder interferir, no momento certo, caso notem alguma deficiência no processo; e

- meio ambiente: considera-se que todas as fases de processamento devem evitar degradações do ambiente com pensamento de melhor aproveitamento da matéria-prima, minimizando os resíduos e ruídos.

De acordo com Sulaiman et al. (2009), as propriedades e características da superfície da madeira lixada diferenciam-se muito daquelas obtidas em plainas. Danos mecânicos podem ser provocados pelo processo de lixamento, como a compressão das paredes das células (Stewart \& Crist, 2001). As superfícies lixadas são também caracterizadas pela presença de fibras arrancadas e levantadas pela ação abrasiva.

No trabalho realizado por Hernández \& Cool (2008b), imagens microscópicas de superfícies preparadas com lixa e plaina foram analisadas. $\mathrm{Na}$ superfície lixada, foram observadas ranhuras típicas, produzidas pela ação do grão da lixa. Além disso, os lumes das células foram preenchidos pelo pó produzido pelo processo de lixamento, o qual dificulta a penetração de adesivos e produtos de acabamento. Em relação à superfície aplainada, os autores relataram que as células apresentaram pequenos danos, com relativamente maior quantidade de células abertas, como vasos e fibras.

De acordo com Muenchow (2002), a superfície a ser colada deve estar no esquadro e, quando se optar pelo lixamento na preparação da superfície das peças, o mesmo autor recomenda utilizar lixa grão 50 a 70. Lixas com grãos mais finos podem ocasionar superfícies polidas, ou vitrificadas, as quais dificultam a penetração do adesivo na madeira. Sulaiman et al. (2009) citam também que, no processo de lixamento, além das características da madeira, o número do grão e o tipo de abrasivo são importantes para a rugosidade. Em geral, quando o número da lixa aumenta, a rugosidade diminui (Burdurlu et al., 2005).

Isso pode ser comprovado pelo trabalho de Kilic et al. (2006), no qual estudou-se o efeito de várias técnicas de usinagem na rugosidade da superfície de Fagus orientalis e Populus tremula. Nenhuma diferença estatística foi observada entre as características da rugosidade das superfícies tangencial e radial. Os autores observaram também que, para ambas as espécies, a rugosidade das amostras melhorou significativamente com o aumento do número do grão da lixa.

No estudo de Burdurlu et al. (2005), foram avaliados o efeito de diferentes números de facas e diferentes grãos de lixas na rugosidade da superfície de madeiras obtidas de Populus nigra e Pinus nigra. Diferentemente de Kilic et al. (2006), os autores observaram uma diferença significativa entre a face radial e tangencial da madeira. A usinagem na face tangencial produziu uma superfície mais lisa que na face tangencial.

Ainda sobre o mesmo estudo, quando foi avaliado somente o efeito da preparação da superfície, os autores perceberam que houve diferença significativa para a rugosidade entre as diferentes lixas usadas. A diferença também foi significativa entre as superfícies preparadas com lixa daquelas preparadas com plaina. Já quando as superfícies preparadas com diferentes números de facas foram comparadas, não foi observado diferença.

Outra observação feita pelos autores corrobora com o resultado de Kilic et al. (2006). Com o aumento do número da lixa, superfícies menos rugosas foram obtidas, no entanto, o lixamento com a lixa 180 produziu uma superfície mais lisa comparada com a superfície lixada com lixa 220.

Como citado anteriormente, são muitos os equipamentos disponíveis para a medição da rugosidade superficial, cada um com suas vantagens e desvantagens. $\mathrm{O}$ método com agulha (haste) tem sido muito usado para determinar a rugosidade da superfície de madeira sólida e compósitos de madeira, apresentando bons resultados (Silva et al., 2006; Varanda et al. 2010; Tiburcio, 2009; Braga et al., 2010; Silva et al., 2008; Kilic et al., 2006; Burdurlu et al., 2005; Hiziroglu et al., 2004; Ozdemir \& Hiziroglu, 2007; Sulaiman et al., 2009). Uma das principais vantagens desse método é a possibilidade de obter o real perfil da rugosidade da superfície e os parâmetros numéricos padrão, que podem ser calculados a partir do perfil. 
Segundo Silva et al. (2008), os parâmetros de rugosidade representam uma forma da expressão matemática entre a variação vertical das superfícies, expressa pelos picos e vales, e o deslocamento horizontal do sensor (agulha). Segundo a norma JIS 0601 (Japanese..., 2001), os parâmetros que definem a rugosidade são: $\mathrm{R}_{\mathrm{a}}$, média aritmética dos valores absolutos dos desvios do perfil da linha média; $\mathrm{R}_{\mathrm{z}}$, soma da altura média dos cinco picos mais altos do perfil e a profundidade dos cinco vales mais profundos do perfil, medidos de uma linha paralela à linha média; $\mathrm{R}_{\mathrm{t}}$ a soma da altura do pico máximo do perfil e a profundidade do vale máximo do perfil sobre a extensão avaliada; $\mathrm{R}_{\mathrm{q}}$, raiz quadrada da média aritmética dos quadrados dos desvios do perfil a partir da linha média dentro do percurso de medição.

Nesse contexto, o objetivo do presente trabalho foi analisar o efeito da usinagem feita por plaina e lixa na rugosidade da superfície da madeira de E. benthamii.

\section{MATERIAL E MÉTODOS}

O material utilizado para a pesquisa foi fornecido pela empresa Klabin S.A. e é proveniente de área de plantio experimental no município de Otacílio Costa, Santa Catarina. A área de coleta do material foi implantada no ano de 1995, com sementes de procedência geográfica de Dorrigo - Austrália, e o espaçamento do povoamento era de 2,5 × 3,0 m. No momento do corte, as árvores apresentavam 12 anos de idade.

Foram abatidas 50 árvores de E. benthamii. Dessas, foram utilizadas as primeiras toras para o processo de desdobro, as quais apresentavam em média três metros de comprimento. As toras apresentaram duas distribuições diamétricas, sendo as toras mais finas com DAP de 20 a $25 \mathrm{~cm}$ e as toras mais grossas com DAP de 26 a $30 \mathrm{~cm}$. Após o processo de desdobro, tábuas de face tangencial e radial foram submetidas à secagem em secador piloto laboratorial no Laboratório de Secagem do Centro de Ciências Exatas e Tecnológicas da Uniplac, até umidade final de aproximadamente 12\%. Destas, foram selecionadas 30 tábuas de face tangencial com dimensões de $3 \mathrm{~m}$ de comprimento, $40 \mathrm{~mm}$ de espessura e larguras variáveis, as quais foram selecionadas visualmente por apresentarem menores defeitos de secagem como empenamentos e rachaduras. O material foi transportado para o Laboratório de Produtos Florestais (Serviço Florestal Brasileiro), onde permaneceu sob climatização (temperatura de $20 \pm 2{ }^{\circ} \mathrm{C}$ e umidade relativa de $65 \pm 2 \%$ ) por um período de aproximadamente 6 meses, quando se deu o início dos trabalhos.

Para a medição da rugosidade, foram cortadas peças nas dimensões de $700 \mathrm{~mm}$ de comprimento, $55 \mathrm{~mm}$ de largura e $25 \mathrm{~mm}$ de espessura. A superfície lixada foi obtida através do lixamento manual, obedecendo a um procedimento padrão. Deste modo, as peças foram friccionadas por 40 vezes, no sentido paralelo à grã da madeira, sobre uma lixa de aproximadamente $1,5 \mathrm{~m}$ de comprimento fixada sobre uma bancada. A lixa de grão 60 era composta de costado de papel e abrasivo de óxido de alumínio. Para preparar a superfície aplainada, foi utilizada uma plaina desempenadeira com 2 facas (Invicta) com rotação do eixo porta-facas de $3440 \mathrm{rpm}$. A profundidade do corte da plaina foi de aproximadamente $0,6 \mathrm{~mm}$.

A medição da rugosidade da superfície foi feita usando o rugosímetro Surftest SJ - 301 (Mitutoyo..., 2004), conforme Figura 1. O aparelho foi programado para medir a rugosidade da superfície de acordo com a norma JIS B 0601 (Japanese..., 2001), com cut-off de $2,5 \mathrm{~mm}$ e o percurso de avaliação de $12,5 \mathrm{~mm}$.

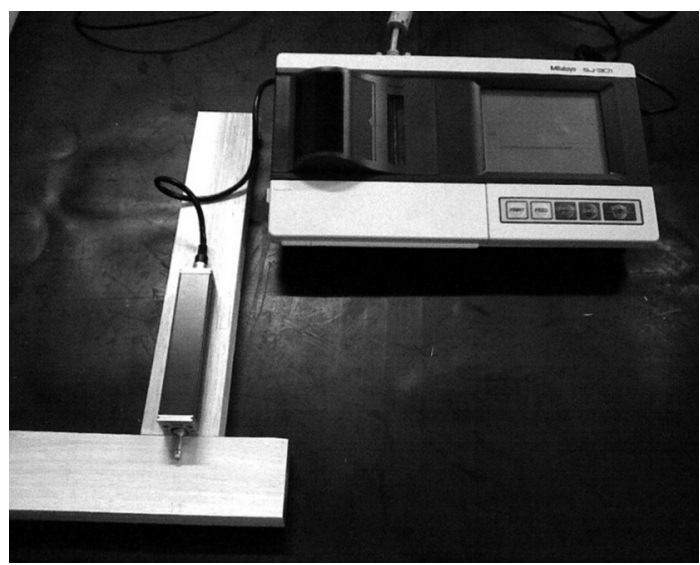

Figura 1. Rugosímetro utilizado para determinar os parâmetros de rugosidade das superfícies aplainadas e lixadas de E. benthmii.

Figure 1. Profilometer utilized to determine the roughness parameters of the planed and sanded E. benthamii surface. 
Foram analisados os parâmetros $R_{a}, R_{z}$ e $R_{t}$ por serem os parâmetros usualmente estudados por outros autores (Sulaiman et al., 2009; Ozdemir \& Hiziroglu, 2007; Hiziroglu et al., 2004), sendo que muitos avaliam apenas o parâmetro $\mathrm{R}_{\mathrm{a}}$ (Burdulu et al., 2005; Moura \& Hernández, 2006). Os parâmetros $\mathrm{R}_{\mathrm{a}}, \mathrm{R}_{\mathrm{z}}$ e $\mathrm{R}_{\mathrm{t}}$ são determinados de acordo com as Equações 1, 2 e 3, respectivamente (Mitutoyo Corporation, 2004).

$$
\begin{aligned}
& \mathrm{Ra}=1 / \mathrm{N} \sum_{\mathrm{i}=1}^{\mathrm{N}}|\mathrm{Yi}| \\
& \mathrm{Rz}=1 / 5 \sum_{\mathrm{i}=1}^{5} \mathrm{Ypi}+1 / 5 \sum_{\mathrm{i}=1}^{5} \mathrm{Yvi} \\
& \mathrm{Rt}=\mathrm{Yp}+\mathrm{Yv}
\end{aligned}
$$

em que: $\mathrm{Yi}=$ Desvios do perfil; $\mathrm{Ypi}=$ Picos mais altos; Yvi = Vales mais profundos; $\mathrm{Yp}=$ Pico mais alto; $\mathrm{Yv}=$ Vale mais profundo.

Foram utilizadas 10 amostras, sendo 5 peças lixadas e 5 peças aplainadas e, de cada peça, foram feitas 2 medições no sentido transversal às fibras, de modo que as ranhuras produzidas pela lixa fossem identificadas pela agulha. Os resultados foram analisados por meio da média dos parâmetros da rugosidade da superfície obtida pelos tratamentos. Para a análise dos resultados, foi empregada a análise de variância (ANOVA) para observar a diferença entre as médias dos tratamentos. As análises foram realizadas com o auxílio do Programa SPSS (Statistical Package for Social Sciences) versão 17.0.

Para a análise de imagens, foram retiradas pequenas amostras de $5 \mathrm{~cm}$ de espessura, $5 \mathrm{~cm}$ de largura e $5 \mathrm{~cm}$ de comprimento, das peças preparadas anteriormente. Sendo obtidas imagens com um aumento de 50x das superfícies lixadas e aplainadas. Para visualização das superfícies, foi utilizado o equipamento estereomicroscópio trinocular modelo 2000 C, e a captação das imagens foi feita com o auxílio de uma câmara modelo AxioCam MRc5 de 5 megapixel acoplada ao estereomicroscópio, ambos da marca Carl Zeiss. A análise foi feita no Instituto Nacional de Criminalística (INC) da Polícia Federal, situado em Brasília - DF.

\section{RESULTADOS E DISCUSSÃO}

A Figura 2 apresenta o perfil típico da rugosidade da superfície aplainada e da superfície lixada.

Com base nos gráficos gerados na avaliação da rugosidade, foi observado que os perfis das superfícies aplainadas apresentaram vales mais profundos, bem como a extensão desses vales foi maior. Isso significa que a plaina produziu superfícies com células abertas, provavelmente vasos. Por outro lado, o perfil da superfície lixada foi caracterizado
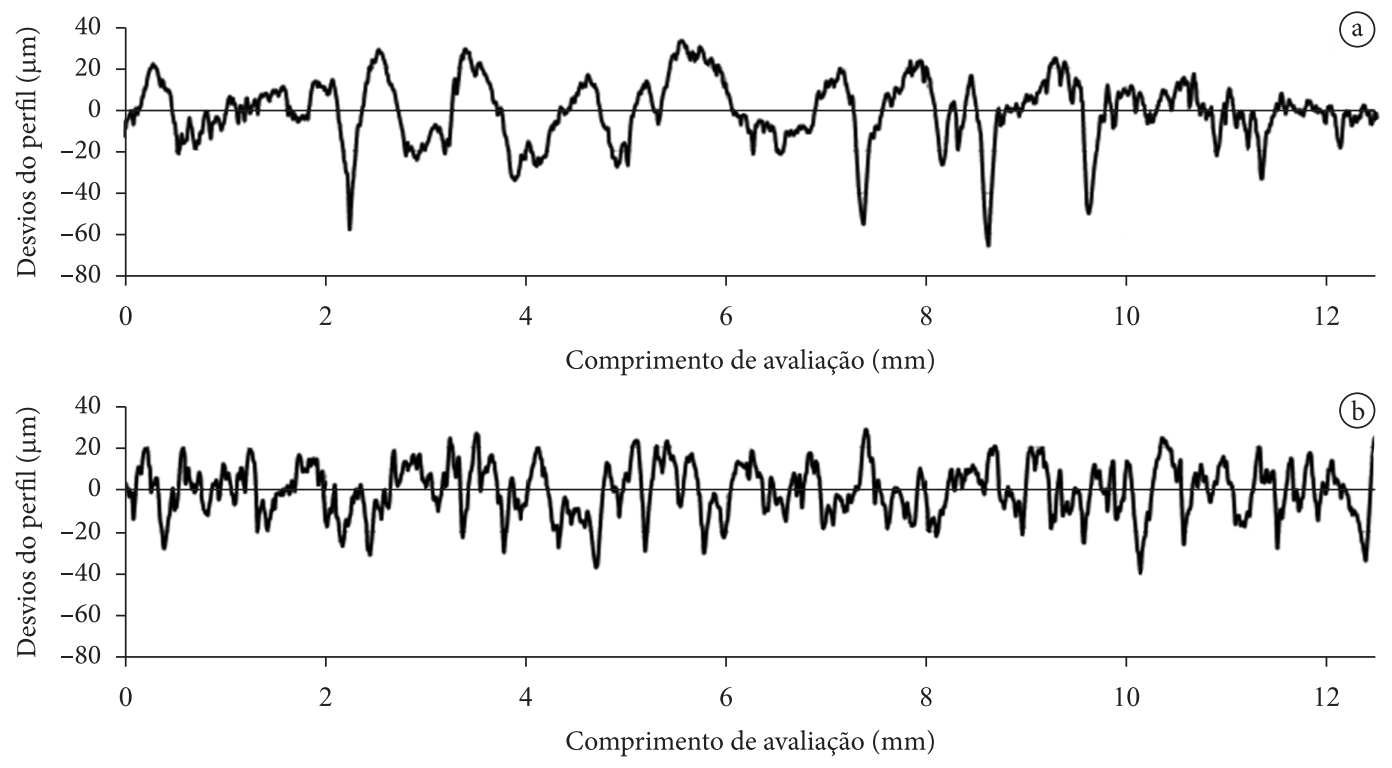

Figura 2. Perfil típico da superfície aplainada (a) e lixada (b) de E. benthamii.

Figure 2. Typical profile of the planed (a) and sanded (b) E. benthamii surface. 
por uma incidência maior de picos e vales menores, correspondendo às ranhuras características produzidas pela lixa. Observações semelhantes foram feitas por Moura \& Hernández (2005), quando analisaram a rugosidade das superfícies aplainadas e lixadas para a aplicação de vernizes.

$\mathrm{Na}$ Tabela 1, são apresentados os valores obtidos para os parâmetros $R_{a}, R_{z}$ e $R_{t}$ para as superfícies preparadas em plaina e em lixa, assim como os respectivos desvios padrão.

Baseado na análise estatística, nenhuma diferença significativa (Tabela 2) foi observada entre a superfície aplainada e a superfície lixada para os parâmetros $\mathrm{R}_{\mathrm{a}}, \mathrm{R}_{\mathrm{z}} \mathrm{e} \mathrm{R}_{\mathrm{t}}$. Porém, em geral foram notados maiores desvios padrão em todos os parâmetros analisados para a superfície aplainada.

$\mathrm{O}$ valor de $\mathrm{R}_{\mathrm{a}}$ encontrado para ambas as superfícies foi maior que o encontrado por Kilic et al. (2006) em superfícies de Populus tremula preparadas com plaina $(7,05 \mu \mathrm{m})$ e lixa grão $60(6,68 \mu \mathrm{m})$ e, também, para a espécie Fagus orientalis nas mesmas condições (plaina - 7,90 $\mu \mathrm{m}$ e lixa grão 60 - 8,20 $\mu \mathrm{m}$ ). Quanto aos valores de $\mathrm{R}_{\mathrm{z}}$, os autores obtiveram em superfícies aplainadas 61,62 e 63,45 $\mu \mathrm{m}$ para Populus tremula e Fagus orientalis, respectivamente. Esses ficaram acima do encontrado para E. benthamii. Enquanto na superfície lixada (grão 60), o parâmetro $\mathrm{R}_{\mathrm{z}}$ do E. benthamii foi intermediário ao Populus tremula $(58,77 \mu \mathrm{m})$ e Fagus orientalis $(68,33 \mu \mathrm{m})$ nas mesmas condições.

Quando comparado com os resultados obtidos por Burdurlu et al. (2005), o valor de $\mathrm{R}_{\mathrm{a}}$ da superfície lixada foi menor que o encontrado para Populus nigra e Pinus nigra lixadas com lixa grão 60, 15,84 e $12,27 \mu \mathrm{m}$, respectivamente. Por outro lado, para a superfície aplainada o valor de $\mathrm{R}_{\mathrm{a}}$ foi maior que o encontrado por aqueles autores. $\mathrm{O}$ parâmetro $\mathrm{R}_{\mathrm{a}}$ para Populus nigra foi $8,85 \mu \mathrm{m}$, enquanto que para Pinus nigra foi $4,51 \mu \mathrm{m}$.

Uma análise das imagens obtidas mostra que, na superfície lixada, podem ser visualizadas fibrilações (Figura 3a) e ranhuras (Figura 3b) causadas pela ação abrasiva do lixamento, particularidades que não foram observadas na superfície aplainada.

A fibrilação apresentada pela superfície lixada pode ser desejável para adesão, mas deve ser limitada a um nível que não possa ser considerado um defeito, como por exemplo, fibra áspera - fuzzy grain (Hernández \& Cool, 2008a). Em relação às ranhuras, essas auxiliam na colagem por oferecem melhores condições de espalhamento do adesivo, já que aceleram a condução de líquidos paralela à grã da madeira (Moura \& Hernández, 2005).

Fendas relativas às células de vasos (Figura $4 \mathrm{a}$ ) ficaram evidentes nas superfícies aplainadas, as quais promovem maior possibilidade para penetração

Tabela 1. Valores dos parâmetros de rugosidade encontrados no teste de rugosidade.

Table 1. Values of the parameters test roughness.

\begin{tabular}{lccc} 
Tratamento & $\mathbf{R}_{\mathbf{a}}(\boldsymbol{\mu m})$ & $\mathbf{R}_{\mathbf{z}}(\boldsymbol{\mu m})$ & $\mathbf{R}_{\mathbf{t}}(\boldsymbol{\mu m})$ \\
\multirow{2}{*}{ Plaina } & 9,08 & 60,16 & 81,96 \\
& $(1,62)$ & $(11,50)$ & $(18,54)$ \\
\multirow{2}{*}{ Lixa } & 9,34 & 59,79 & 76,99 \\
& $(0,41)$ & $(5,78)$ & $(9,84)$ \\
\hline
\end{tabular}

Valores entre parênteses correspondem ao desvio padrão.

Tabela 2. Análise de variância (ANOVA) para os parâmetros de rugosidade das superfícies aplainadas e lixadas. Table 2. Analysis of variance (ANOVA) for roughness parameters of planed and sanded surfaces.

\begin{tabular}{rcccccc}
$\begin{array}{r}\text { Fonte de } \\
\text { variação }\end{array}$ & Propriedade & $\begin{array}{c}\text { Soma dos } \\
\text { quadrados }\end{array}$ & $\begin{array}{c}\text { Graus de } \\
\text { liberdade }\end{array}$ & $\begin{array}{c}\text { Quadrado } \\
\text { médio }\end{array}$ & F calc & Significância \\
\hline \multirow{2}{*}{ Tratamento } & $\mathrm{R}_{\mathrm{a}}$ & 0,320 & 1 & 0,320 & 0,218 & $0,647^{\mathrm{NS}}$ \\
& $\mathrm{R}_{\mathrm{z}}$ & 0,703 & 1 & 0,703 & 0,008 & $0,928^{\mathrm{NS}}$ \\
& $\mathrm{R}_{\mathrm{t}}$ & 123,405 & 1 & 123,405 & 0,560 & $0,464^{\mathrm{NS}}$ \\
\hline
\end{tabular}

${ }^{\mathrm{N} S}$ diferença entre os valores de plaina e lixa não significativa. 

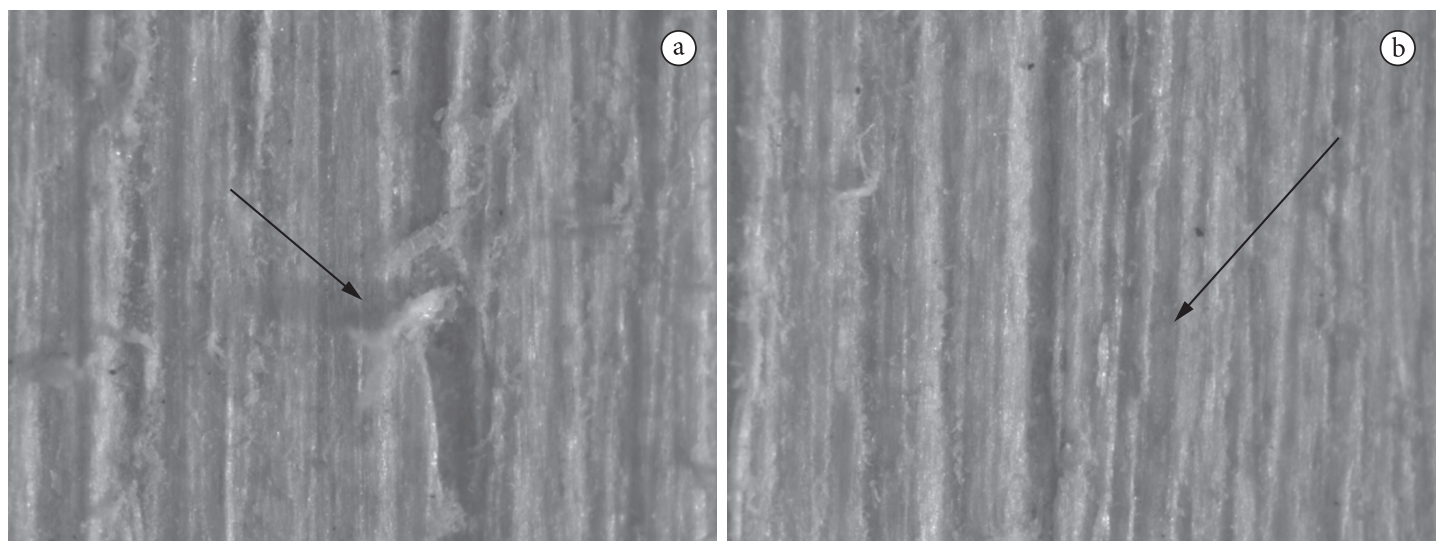

Figura 3. Imagem da superfície lixada de Eucalyptus benthamii (50x) com a presença de fibrilações (a) e ranhuras (b). Figure 3. Sanded surface of Eucalyptus benthamii image (50x) with fibrillation (a) and scratches (b).
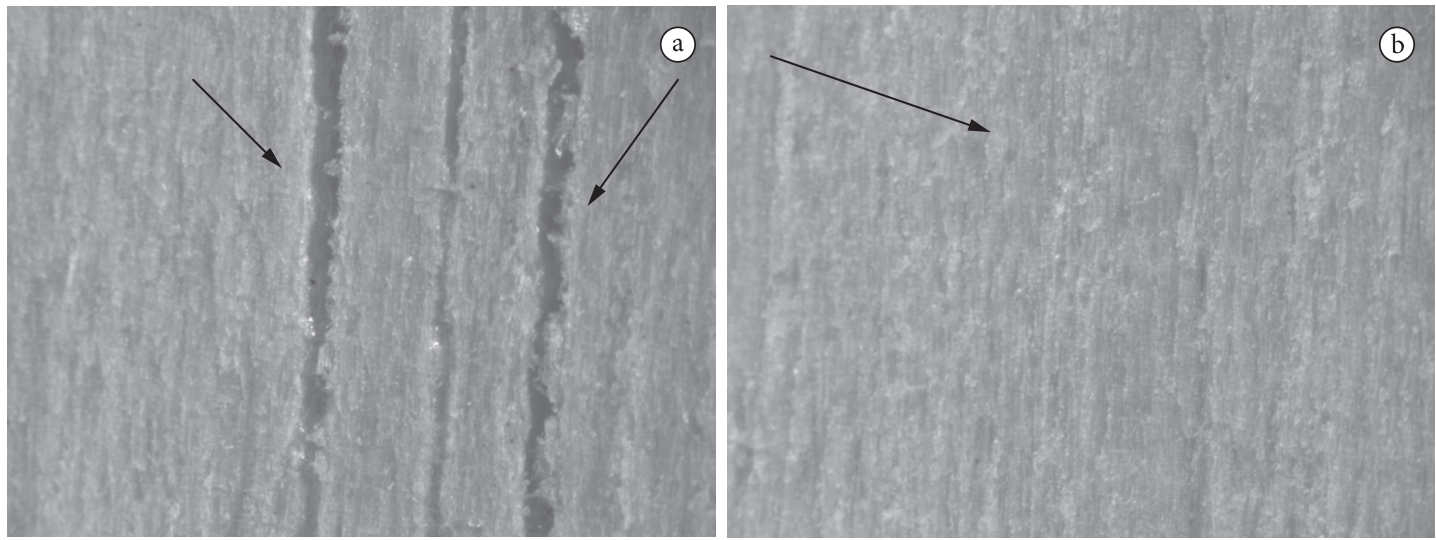

Figura 4. Imagem da superfície aplainada de Eucalyptus benthamii (50x) com presença de células de vasos abertas (a) e sem ranhuras (b).

Figure 4. Planed surface of Eucalyptus benthamii image (50x) with open vessel cells (a) and no scratches (b).

do adesivo, auxiliando na colagem de madeira (Moura \& Hernández, 2005). Além disso, percebese visualmente maior lisura da superfície em comparação à superfície lixada (Figura 4b).

Análises similares foram feitas por Moura \& Hernández (2005), e Hernández \& Cool (2008b), quando avaliaram através de imagens, superfícies lixadas e aplainadas de Acer saccharum e Betula papyrifera, respectivamente.

\section{CONCLUSÕES}

Pode-se concluir que os parâmetros de rugosidade $\mathrm{R}_{\mathrm{a}}, \mathrm{R}_{\mathrm{z}}$ e $\mathrm{R}_{\mathrm{t}}$ da superfície de Eucalyptus benthamii não foram influenciados pelo modo de processamento da superfície (plaina e lixa). Entretanto, através da análise de imagem foi observado que a superfície lixada apresentou fibrilações e ranhuras, enquanto a superfície aplainada apresentou células de vasos abertas.

\section{AGRADECIMENTOS}

Ao $\mathrm{CNPq}$ pela bolsa de mestrado em Ciências Florestais concedida ao primeiro autor, à Klabin Unidade Otacílio Costa - SC pelo material lenhoso fornecido e à Polícia Federal de Brasília por disponibilizar o uso do estereomicroscópio trinocular. 


\section{STATUS DA SUBMISSÃO}

Recebido: 14/12/2010

Aceito: 06/04/2011

Resumo publicado online: 13/04/2011

Artigo completo publicado: 30/06/2011

\section{AUTOR(ES) PARA CORRESPONDÊNCIA}

\section{Sabrina Andrade Martins}

Departamento de Engenharia Florestal, Faculdade de Tecnologia, Universidade de Brasília - UnB, Campus Darcy Ribeiro, CP 04357, CEP 70919-970, Brasília, DF, Brasil e-mail: sabrinaand@gmail.com

\section{REFERÊNCIAS}

Bonduelle A, Cruz CR, Silva JRM. Processo mecânico da madeira. Curitiba: UFPR, 2002. Notas de aula.

Braga PPC, Silva JRM, Gontijo TG, Teixeira RL, Nunes ACM. Qualificação de superfícies de diferentes madeiras usinadas. In: Anais Simpósio de Pós-Graduação em Ciências Florestais; 6.; Simpósio de Ciência e Tecnologia da Madeira do Rio de Janeiro; 2.; 2010; Rio de Janeiro. Rio de Janeiro: IF, 2010.

Burdurlu E, Usta I, Ulupinar M, Aksu B, Erarslan Ç. The effect of the number of blades and grain size of abrasives in planing and sanding on the surfasse roughness of European black pine and Lombardy poplar. Turkish Journal of Agriculture \& Forestry 2005; 25:315-321.

Hernández RE, Cool J. Effects of cutting parameters on surfasse quality of paper birch wood machined across the grain with two planing techniques. Holz als Roh und Werkstoff 2008a; 66:147-154. http://dx.doi.org/10.1007/ s00107-007-0222-4

Hernández RE, Cool J. Evaluation of three surfacing methods on paper birch wood in relation to water-and solvent-borne coating performance. Wood and Fiber Science 2008b; 40(3):459-469.

Higa AR, Carvalho PER. Sobrevivência e crescimento de doze espécies de eucalipto em Dois Vizinhos, Paraná. Silvicultura 1990; (42):459-461. Edição dos Anais do $6^{\circ}$ Congresso Florestal Brasileiro; 1990; Campos do Jordão.

Higa RCV, Pereira JCD. Usos Potenciais do Eucalyptus benthammii Maiden et Cambage. Colombo: Embrapa Florestas, 2003. 4 p. (Comunicado Técnico).

Hiziroglu S, Jarusombuti S, Fueangvivat V. Surface characteristics of wood composites manufactured in
Thailand. Building and Environment 2004; 39:1359-1364. http://dx.doi.org/10.1016/j.buildenv.2004.02.004

Japanese Industrial Standard - JIS. JIS B 0601:2001: Geometrical Products Specifications (GPS) - Surface texture: Profile method - Terms, definitions and surface texture parameters. Tokyo: JIS; 2001.

Kilic M, Hiziroglu S, Burdurlu E. Effect of machining on surface roughness of wood. Building and Environment 2006; 41(8):1074-1078. http://dx.doi.org/10.1016/j. buildenv.2005.05.008

Mitutoyo Corporation. SJ - 301 Surface Roughness Tester: User's manual. 2004. 346p.

Moura LF, Hernández RE. Effects of abrasive mineral, grit size and feed speed on the quality of sanded surfaces of sugar maple wood. Wood Science and Technology 2006; 40:517-530. http://dx.doi.org/10.1007/s00226006-0070-0

Moura LF, Hernández RE. Evaluation of varnish coating performance for two surfacing methods on sugar maple wood. Wood and Fiber Science 2005; 37(2):355-366.

Muenchow J. Os passos do processo de colagem lateral e colagem por face em alta frequência. Franklin International; 2002.9 p. (Material técnico).

Nisgoski S, Muñiz GIB, Klock U. Caracterização anatômica da madeira de Eucalyptus benthamii Maiden et Cambage. Ciência Florestal 1998; 8(1)67-76.

Ozdemir T, Hiziroglu S. Evaluation of surface quality and adhesion strength of treated solid wood. Journal of Materials Processing Technology 2007; 186:311-314. http://dx.doi.org/10.1016/j.jmatprotec.2006.12.049

Pereira JCD, Schaitza EG, Shimizu J. Características físicas, químicas e rendimento da destilação seca da madeira de Eucalyptus benthamii. Curitiba: Embrapa Florestas; 1997. 2 p. (Relatório de Pesquisa em Andamento; n. 35).

Piao C, Winandy JE, Shupe TF. From hydrophilicity to hydrophobicity: a critical review: Part 1 . Wettability and surface behavior. Wood and Fiber Science 2010; 42(4):490-510.

River BH, Vick CB, Gillespie RH. Wood as an adherend. In: Minford J. Treatise on Adhesion and Adhesives, New York: J. Dean Minford; 1991. vol. 7, 230 p.

Silva JRM, Braga PPC, Martins M, Filipe AP. Identificação de parâmetros de rugosidade para qualificação de pisos de Eucalyptus grandis. In: Anais Encontro Brasileiro em Madeira e Estruturas de Madeira; 11.; 2008; Londrina. Londrina: Universidade Estadual de Londrina; 2008.

Silva JRM, Lima JT, Braga PPC, Trugilho PF. A utilização de rugosímetro na qualificação de superfícies usinadas em madeiras de Eucalyptus sp. In: Anais Encontro Brasileiro em Madeira e Estruturas de Madeira; 10.; São Pedro. São Pedro: UNESP; 2006. 
Stewart HA, Crist JB. SEM examination of subsurface damage of wood after abrasive and knife planing. Wood Science and Technology 2001; 14(3):106-109.

Sulaiman O, Hashim R, Subari K, Liang CK. Effect of sanding on surface roughness of rubberwood. Journal of Materials Processing Technology 2009; 209(8):3949-3955. http://dx.doi.org/10.1016/j.jmatprotec.2008.09.009

Taylor JB, Carrano AL, Lemaster RL. Quantification of process parameters in a wood sanding operation. Forest Products Journal 1999; 49(5):41-46.
Tiburcio UFO. Medição e análise do acabamento superficial da madeira de eucalipto na usinagem de torneamento cilíndrico e lixamento [tese] Guaratingueta: Universidade Estadual Paulista; 2009.

Varanda LD, Alves MCS, Gonçalves MTT, Santiago LFF. A influência das variáveis do lixamento tubular na qualidade das peças de Eucalyptus grandis. Cerne 2010; 16:23-32. 